\title{
Custom Scan Control and Time Resolved Signal Acquisition for High Resolution SEM Imaging
}

William C. Lenthe ${ }^{1,3}$, Jean-Charles Stinville ${ }^{1}$, McLean P. Echlin ${ }^{1}$, Zhe Chen ${ }^{2}$, Samantha Daly ${ }^{2}$, Tresa M. Pollock ${ }^{1}$, Marc De Graef ${ }^{3}$,

1. Dept. of Materials, University of California at Santa Barbara, Santa Barbara, CA, USA

2. Dept. of Mechanical Engr., University of California at Santa Barbara, Santa Barbara, USA

3. Dept. of Materials Science and Engineering, Carnegie Mellon Univ., Pittsburgh, PA, USA

Drift, electron lens quality, and other sources of SEM artifacts limit imaging resolution and are difficult to completely mitigate experimentally $[1,2]$. The capacity for modern SEMs to accommodate load frames, heating/cooling stages, and other in-situ instrumentation continues to advance materials science at the mesoscale but can also reveal or exacerbate these equipment limitations [3,4]. The development of high resolution SEM DIC for measurement of elastic and plastic strains has enabled quantification of and correction for scan field distortions and beam scanning defects [5-7]. In this study, time resolved signal acquisition enables measurement and correction of beam scanning defects.

SEM images were collected using high speed (2 million samples per second) data acquisition. Individual samples were maintained as a vector of time resolved detector responses for each pixel instead of an integrated measurement, making each pixel a drift experiment. A virtual image reconstructed from the $n^{\text {th }}$ sample of each pixel reveals an apparent shift as shown in Fig. 1. The shift for each sample is efficiently computed by maximizing cross correlation with respect to the final sample [8]. Correcting for these shifts before integrating results in images with improved spatial resolution. Time resolved images have been collected over a range of electron column conditions and using a variety of scan patterns, electron detectors, and electron beam deflectors with response time curves shown in Fig. 2. Measured beam settling times are long ( $5 \mu \mathrm{s}$ to $15 \mu \mathrm{s})$ compared to typical dwell times for SE and BSE imaging [9].

References:

[1] G Nolze, Ultramicroscopy 107 (2007), p. 172-183.

[2] MA Sutton et al, Meas. Sci. Technol. 17 (2006), p. 2613.

[3] JL Carter et al, Mater. Sci. Eng., A 605 (2014), p. 127-136.

[4] TJ Woehl et al, Ultramicroscopy 127 (2013), p. 53-63.

[5] MA Sutton et al, Exp. Mech. 47 (2007), p. 775-787.

[6] MA Sutton et al, Exp. Mech. 47 (2007), p. 789-804.

[7] AD Kammers and S Daly, Exp. Mech. 53 (2013), p. 1333-1341.

[8] M Guizar-Sicairos, ST Thurman, and JR Fienup, Opt. Lett. 33(2) (2008) pp. 156-158.

[9] The authors acknowledge ONR Vannevar Bush Faculty Fellowship support (N00014-16-1-2821) as well as ONR grant N00014-16-1-2982 and an AFRL/AFOSR Center of Excellence Grant (FA9550-121-0445). This work was supported by the U.S. Department of Energy, Office of Basic Energy Sciences, Division of Materials Sciences and Engineering under Award \#DESC0008637 as part of the Center for PRedictive Integrated Structural Materials Science (PRISMS) at the University of Michigan. 




First Sample ( $\mathrm{t}=0 \mu \mathrm{s})$

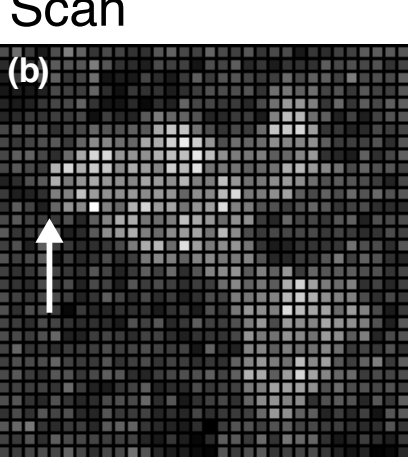

Last Sample ( $\mathrm{t}=19.5 \mu \mathrm{s})$

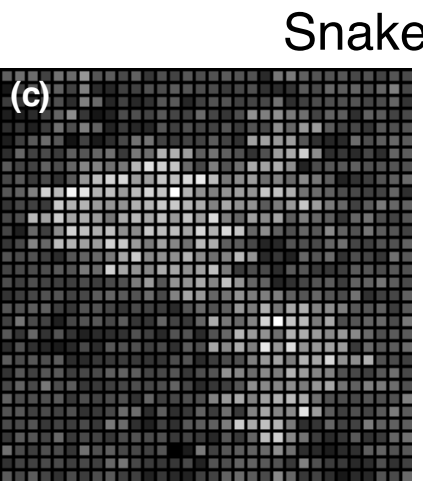

First Sample $(\mathrm{t}=0 \mu \mathrm{s})$

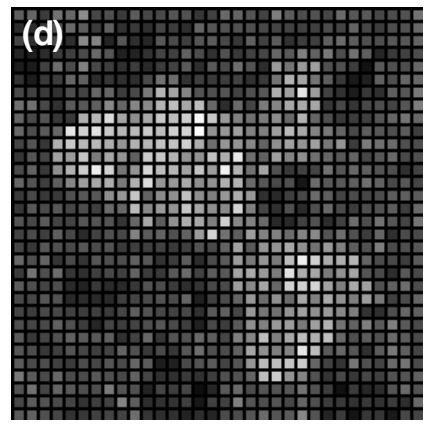

Last Sample ( $\mathrm{t}=19.5 \mu \mathrm{s})$

Figure 1. A $32 \times 32$ pixel sub-region of an SEM image of particles (light) on René 88DT ( $\gamma$ phase medium gray, $\gamma^{\prime}$ phase dark gray) imaged with at $20 \mathrm{kV}$ is shown. The first sample of each pixel (a) is shifted 1 pixel with respect to the final sample of each pixel (b). The result is a subtle rigid shift for a raster scan pattern (left), but is more obvious when the same region is imaged with a snake pattern (c/d).

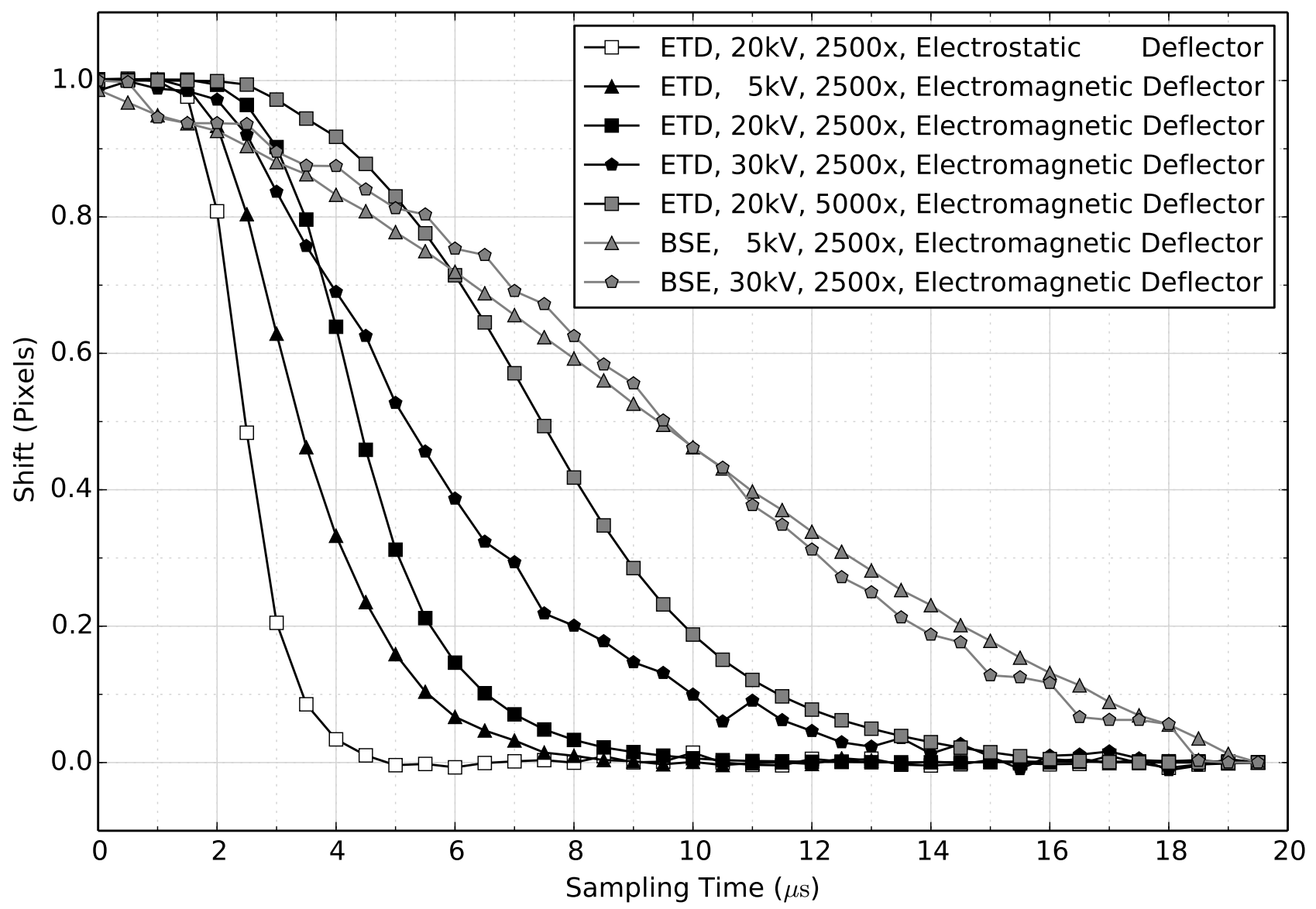

Figure 2. Computed shifts are plotted against sample collection time for a variety of imaging condi-tions. Electrostatic deflectors are more responsive than electromagnetic deflectors. Everhart-Thornley secondary detectors are more responsive than solid state BSE detectors but exhibit an accelerating voltage dependence. Column response time is worse as more lenses engage at higher magnifications. 